\title{
Changes in the Spinal Segmental Motor Output for Stepping during Development from Infant to Adult
}

\author{
Yuri P. Ivanenko, ${ }^{1}$ Nadia Dominici, ${ }^{1,2,3}$ Germana Cappellini, ${ }^{1}$ Ambrogio Di Paolo, ${ }^{4}$ Carlo Giannini, ${ }^{5}$ Richard E. Poppele, ${ }^{6}$ \\ and Francesco Lacquaniti ${ }^{1,2,4}$ \\ ${ }^{1}$ Laboratory of Neuromotor Physiology, IRCCS Santa Lucia Foundation, 00179 Rome, Italy, ${ }^{2}$ Centre of Space Bio-medicine, University of Rome Tor Vergata, \\ 00173 Rome, Italy, ${ }^{3}$ Center for Neuroprosthetics and Brain Mind Institute, School of Life Sciences, Swiss Federal Institute of Technology, 1015 Lausanne, \\ Switzerland, ${ }^{4}$ Department of Systems Medicine, University of Rome Tor Vergata, 00133 Rome, Italy, ${ }^{5}$ Neonatology Unit, Sant'Eugenio Hospital, 00144 \\ Rome, Italy, and ${ }^{6}$ Department of Neuroscience, University of Minnesota, Minneapolis, Minnesota 55455
}

Human stepping movements emerge in utero and show several milestones during development to independent walking. Recently, imaging has become an essential tool for investigating the development and function of pattern generation networks in the spinal cord. Here we examine the development of the spinal segmental output by mapping the distribution of motoneuron activity in the lumbosacral spinal cord during stepping in newborns, toddlers, preschoolers, and adults. Newborn stepping is characterized by an alternating bilateral motor output with only two major components that are active at all lumbosacral levels of the spinal cord. This feature was similar across different cycle durations of neonate stepping. The alternating spinal motor output is consistent with a simpler organization of neuronal networks in neonates. Furthermore, a remarkable feature of newborn stepping is a higher overall activation of lumbar versus sacral segments, consistent with a rostrocaudal excitability gradient. In toddlers, the stance-related motor pool activity migrates to the sacral cord segments, while the lumbar motoneurons are separately activated at touchdown. In the adult, the lumbar and sacral patterns become more dissociated with shorter activation times. We conclude that the development of human locomotion from the neonate to the adult starts from a rostrocaudal excitability gradient and involves a gradual functional reorganization of the pattern generation circuitry.

\section{Introduction}

Spinal central pattern generators (CPGs) contribute to the timing and patterns of coordinated muscle activities during locomotion (Grillner, 1981, 2006; Kiehn, 2006). Comparative studies in vertebrates based on genetic, neurochemical, and electrophysiological approaches demonstrated that, despite species-specific features, there are several common principles in the organization of CPGs (Garcia-Campmany et al., 2010; Kiehn, 2011). Thus, the core premotor components of locomotor circuitry mainly derive from a set of embryonic interneurons that are remarkably conserved across different species (Goulding, 2009). Rhythmogenetic capacity is typically distributed along the lumbosacral cord, with a rostrocaudal excitability gradient (Cazalets and Bertrand, 2000; Lev-Tov et al., 2000; Vinay et al., 2002; Kiehn, 2006). It has also been proposed that the mammalian CPG has two layers: a rhythm-generating layer distinct from a pattern-generating layer (Patla et al., 1985; McCrea and Rybak, 2008). Notably, locomotor

\footnotetext{
Received June 5, 2012; revised Nov. 29, 2012; accepted Dec. 5, 2012.

Author contributions: Y.P.I., N.D., A.D.P., C.G., R.E.P., and F.L. designed research; Y.P.I., N.D., G.C., A.D.P., C.G., and F.L. performed research; Y.P.I., N.D., G.C., R.E.P., and F.L. analyzed data; Y.P.I., N.D., R.E.P., and F.L. wrote the paper.

This work was supported by the Italian Ministry of Health, the Italian Ministry of Education, Universities and Research (PRIN grant), the Italian Space Agency (DCMC and CRUSOE grants) and the European Union FP7-ICT program (AMARSi Grant 248311). We thank Vito Mondi, Marika Cicchese, Adele Fabiano, and Tiziana Silei for help with some of the experiments.

Correspondence should be addressed to Yuri P. Ivanenko, Laboratory of Neuromotor Physiology, IRCCS Santa Lucia Foundation, 00179 Rome, Italy. E-mail: y.ivanenko@hsantalucia.it.

DOI:10.1523/JNEUROSCI.2722-12.2013

Copyright $\odot 2013$ the authors $\quad 0270-6474 / 13 / 333025-12 \$ 15.00 / 0$
}

behaviors involve activation of many muscles. In the spinal cord, motorneurons $(\mathrm{MN})$ are arranged in columns, with a specific grouping of muscles at each segmental level (Romanes, 1951; Sharrard, 1955), and the functional significance of such motorneuron positioning has been debated previously (Cappellini et al., 2010; Jessell et al., 2011).

The neural circuits that govern basic locomotor functions appear to be genetically determined, though the mechanisms leading to early identity and development of sensory-motor and nerve-muscle connectivity remain unclear (Sürmeli et al., 2011). Functional connectivity and transition to coordinated activity of motoneurons emerge with specific timing during development (Lacquaniti et al., 2012b). Thus, optical imaging of spontaneous activity in ventral spinal neurons of the zebrafish embryo show a rapid transition from sporadic slow activity to ipsilaterally correlated and contralaterally anticorrelated fast activity involving several adjacent somites (Warp et al., 2012).

Human locomotion also undergoes changes during development. Muscle activation during infant stepping involve excessive cocontraction of many leg muscles (Forssberg, 1985; Okamoto et al., 2001; Dominici et al., 2011; Teulier et al., 2012), compared to the much more sparse muscle activation of adult walking (Winter and Yack, 1987; Ivanenko et al., 2006). Infant stepping also shares features with stepping in other mammals (Forssberg, 1985; Patrick et al., 2009), and it has been proposed that their control patterns may be similar (Yang and Gorassini, 2006; Dominici et al., 2011). While imaging the spatiotemporal organization of neural activity in the developing spinal cord has received atten- 
tion in previous studies on animals (O'Donovan et al., 2008; Warp et al., 2012), these data are essentially absent for humans.

Here, we examine stepping in newborn infants, using both kinematic and electromyographic recordings. We used the recordings from 12 muscles in each leg to reconstruct the patterns of segmental motor output in the infant lumbosacral spinal cord, and then compared the spatiotemporal activation patterns to those in toddlers, older children, and adults. The data on the segmental nerve supply, together with the methods of mapping EMG activity to the spinal motor pools (Yakovenko et al., 2002; Grasso et al., 2004; Ivanenko et al., 2006), provide a noninvasive tool for investigating the development of the spinal segmental output in humans.

\section{Materials and Methods}

\section{Subjects and protocol}

Neonate stepping was recorded at the well-baby maternity ward of the Sant'Eugenio Hospital, whereas toddler, child, and adult walking was recorded at the Santa Lucia Institute in Rome. The procedures and experiments were approved by the Health Ethics Committees of the Sant'Eugenio Hospital and Santa Lucia Hospital, and conformed to the Declaration of Helsinki for experiments on humans. Informed consent was obtained from the adults and from the parents of the children.

Stepping behavior was observed in 40 of the 47 healthy term neonates. Most of these recordings have been included in a brief report (Dominici et al., 2011), but the spatiotemporal dynamics of motoneuron activation in the lumbosacral spinal cord with the topographical maps were not characterized in the previous report. The characteristics of these 40 infants were as follows: $3.2 \pm 1.4$ days old (mean \pm SD); range, $2-7$ days old; the majority were $2-3$ days old (75\%); 25 males, 15 females; $50.8 \pm$ $2.5 \mathrm{~cm}$ body height; and $3.11 \pm 0.52 \mathrm{~kg}$ body weight. To elicit stepping, infants were held under their arms with their feet touching the horizontal flat walkway surface (see Fig. $2 A$ ). Stepping was typically successful when the infants were not drowsy. The infants were allowed to support as much of their own weight as possible, the rest being supported by the investigator (hospital pediatrician) holding the infant. The infants stepped along a $1 \mathrm{~m}$ walkway. The environment in which the experiments took place (hospital nursery) was appropriate for the neonates.

We separately considered the data of a subset of 10 neonates $(3.7 \pm 1.8$ days old; six males, four females; $50.0 \pm 2.4 \mathrm{~cm}$ body height; $3.13 \pm 0.57$ $\mathrm{kg}$ body weight) extracted from the whole group because they had the largest number of recorded muscles ( $\geq 16$; see Data recording, below). The EMG data of these 10 babies were used for reconstructing the spinal maps of motoneuronal activation and for comparison with the data of the three groups of older subjects ( $n=10$ in each group; see below). Since each neonate produced only a limited number of steps and a limited variety of cycle durations, we included all 40 neonates to characterize overall effects of walking speed on muscle activity. To this end, the pooled EMG data of the 40 neonates were averaged across different ranges of stepping cycle durations.

We compared neonate stepping with normal walking in toddlers who were just beginning to walk independently (within 2 weeks after the onset of unsupported walking; $n=10 ; 11-14$ months; five males, five females), preschoolers (aged 22 to 48 months; $n=10$; seven males, three females), and adults (aged 25 to 40 years; $n=10$; seven males, three females). The subjects were asked to walk along an $8 \mathrm{~m}$ walkway at a natural selfselected speed (on average, $1.9 \pm 0.5 \mathrm{~km} / \mathrm{h}$ in toddlers; $2.8 \pm 0.7 \mathrm{~km} / \mathrm{h}$ in preschoolers; $4.6 \pm 0.6 \mathrm{~km} / \mathrm{h}$ in adults). Ten to 15 steps were typically recorded in each subject.

\section{Data recording}

In neonates, we recorded limb kinematics, vertical foot pressure, and bilateral EMG activity, with all recordings synchronized. The kinematics in the sagittal plane was recorded with a video camera at 25 frames per second. Four markers (12 mm diameter) were placed over the hip (greater trochanter), knee (lateral femur epicondyle), ankle (lateral malleolus), and fifth metatarsophalangeal joint of the right leg (see Fig. 2A). Foot pressure was recorded at $50 \mathrm{~Hz}$ by means of the Tekscan walkway
( $44 \times 95 \mathrm{~cm}$ mat, four sensors per square centimeter) specially calibrated for low pressure values in infants.

The EMG data were recorded bilaterally at $2000 \mathrm{~Hz}$ using the wireless Zerowire system. The skin was cleaned and rubbed slightly with alcohol before application of the electrodes. Generally, we used miniature $(2 \mathrm{~mm}$ recording diameter, to minimize cross talk), $\mathrm{Ag}-\mathrm{AgCl}$, reusable, surface EMG disc electrodes (Beckman Instruments). In few instances (based on the pediatrician's recommendation), we used disposable surface electrodes ( $15 \mathrm{~mm}$; Ambu). To minimize potential movement artifacts, preamplified EMG sensor units were attached on the experimenter wrist (see Fig. 2A), and twisted pairs of wires (between electrodes and units) were limited to $25 \mathrm{~cm}$ length and fixed along the infant leg using elastic gauze. The recording system bandwidth was $20-1000 \mathrm{~Hz}$ with an overall gain of 1000 .

Each recording consisted of several trials and typically lasted 1-2 min, after which the infants rested. During these rest periods, EMGs were often monitored, to determine whether clear and separate bursts of EMG could be obtained from each muscle group (Yang et al., 1998). This is important, as cross talk between antagonist muscles is more likely to occur in infants than adults because of their small size. Quiet periods during the sequence of recording also allowed us to estimate clean baselines and the noise level in each channel. Because of the low skin impedance at each electrode locus and the short length of the twisted wires, typically no artifact or noise was present. While the number of simultaneously recorded muscles was limited to 22 , nevertheless, by recording slightly different sets of muscles in different infants we obtained the whole data set of 28 bilateral EMGs: tibialis anterior (TA), gastrocnemius lateralis (LG), gastrocnemius medialis (MG), soleus (Sol), vastus lateralis (Vlat), vastus medialis (Vmed), rectus femoris (RF), hamstring (HS), adductor longus (Add), tensor fascia latae (TFL), gluteus maximus (GM), erector spinae (ES; recorded at L2), external oblique (OE), and latissimus dorsi (LD). However, the $\mathrm{OE}$ and LD activity in neonates contained mainly electrocardiographic, respiration-related, or cryingrelated activity (see Fig. $3 A$ ) and therefore were not included in the analyses.

In toddlers and adults, we recorded the same set of EMGs as in neonates with the Zerowire system. EMG electrode placement was performed according to recommendations for minimizing cross talk from adjacent muscles (Kendall et al., 1993) and was described in detail previously (Ivanenko et al., 2006). Infrared reflective markers (diameter, 1.4 $\mathrm{cm}$ ) were attached on each side of the subject, and the kinematic data were recorded at $100 \mathrm{~Hz}$ by means of the Vicon 612 system with nine TV cameras spaced around the walkway (Ivanenko et al., 2005b; Dominici et al., 2010).

\section{Data analysis}

Successful sequences of forward stepping were identified off-line from video recordings. In particular, the criteria for choosing the steps for further data analysis were as follows (Yang et al., 1998): alternating (left/ right) foot placements; at least two consecutive strides were performed; and gait initiation and termination steps were excluded from the analysis.

On average, we recorded and analyzed $10 \pm 11$ (range, 4-104 steps) successful steps per infant (356 steps total over all neonates). From the kinematic and contact force recordings, the gait cycle and stride length were defined as the time and distance between two successive foot touchdown events of the right leg.

The mean level of the body weight support (BWS) during infant stepping was computed in each trial from the vertical bilateral contact force recordings $[$ BWS $=$ (mean vertical ground reaction force) $/$ (body weight)]. For illustrative purposes, to characterize the general pattern of foot pressure distribution, peak pressure values of the individual sensors during standing or over the stance phase of walking were displayed using a color scale (see Fig. 2B).

EMG data were high-pass filtered $(40 \mathrm{~Hz})$, rectified, low-pass filtered (zero-lag fourth-order Butterworth filter with cutoff at $10 \mathrm{~Hz}$ ), time interpolated over individual gait cycles to fit a normalized 200-point time base, and averaged (Ivanenko et al., 2005b). In addition, since the cycle duration differed substantially across groups of subjects (see Fig. 2C), the low-pass filter in neonates was normalized relative to that of the adults 
Table 1. Mean counts of limb MNs in the individual segments of the human spinal cord ( $13-40$ years, 12 cases)

\begin{tabular}{cr}
\hline Segment level & \\
L1 & 806 \\
L2 & 5146 \\
L3 & 12765 \\
L4 & 12069 \\
L5 & 12674 \\
S1 & 10372 \\
S2 & 4216 \\
S3 & 409 \\
Total number & 58457 \\
\hline
\end{tabular}

Data are adopted from the study by Tomlinson and Irving (1977).

(i.e., it was made inversely proportional to the cycle duration, corresponding to $3 \mathrm{~Hz}$ in neonates since the cycle duration was about three times longer than in adults) under the assumption that the width of the basic EMG components tends to scale with speed (Ivanenko et al., 2004; Cappellini et al., 2006). Data filtering does not seem to affect the overall shape of the basic temporal components (Ivanenko et al., 2004). Nevertheless, we also performed the same filtering of the EMG data in neonates as in adults ( $10 \mathrm{~Hz}$ low pass) and verified that the results did not change.

Motor output of the spinal segments

Methods of calculation. To characterize the spatiotemporal organization of the total motor output, the recorded averaged patterns of EMG activity were mapped onto the estimated rostrocaudal location of MN pools in the human spinal cord. This approach provides an interpretation of the motor pool activation at a segmental level rather than at the individual muscle level (Yakovenko et al., 2002; Grasso et al., 2004; Ivanenko et al., 2006). It can be used to characterize network architecture for different gaits by considering relative intensities, spatial extent, and temporal structure of the spinal motor output (Ivanenko et al., 2008; Monaco et al., 2010). Comparable functional imaging of MN activity in the human spinal cord remains difficult to apply to walking (Giove et al., 2004; Bosma and Stroman 2012).

Because the method has been thoroughly documented in our previous papers (Grasso et al., 2004; Ivanenko et al., 2006, 2008; Cappellini et al., 2010; Maclellan et al., 2012), here we describe it only briefly. In this study we used the myotomal charts of Kendall et al. (1993). Despite likely anatomical variability both in adults and infants, the data from these charts appear sufficiently robust for the spatial resolution currently available based on EMG recordings from multiple lower limb muscles. In general, each muscle is innervated by several spinal segments, and each segment supplies several muscles. To reconstruct the motor-pool output pattern of any given spinal segment $S_{j}$ of the lumbosacral segments (L2S2) most active during locomotion, all rectified EMG waveforms corresponding to that segment were averaged using appropriate weighting coefficients.

We used two different methods: (1) The nonnormalized method, where EMGs are expressed in microvolts (Ivanenko et al., 2006), is as follows:

$$
S_{j}=\frac{\sum_{i=1}^{n_{j}} k_{i j} \cdot \mathrm{EMG}_{i}}{n_{j}},
$$

where $n_{j}$ is the number of $\mathrm{EMG}_{i} \mathrm{~s}$ corresponding to the $j$ th segment, and $k_{i j}$ is the weighting coefficient for the $i$ th muscle ( $X$ and $x$ in Kendall's chart were weighted with $k_{i j}=1$ and $k_{i j}=0.5$, respectively). (2) For the normalized method, EMGs were expressed in microvolts and normalized to the physiological cross-sectional area (PCSA) of lower limb muscles as determined by Ward et al., (2009). To this end, the contribution of each muscle to the estimated motor pool activity of the $j$ th segment (Eq. 1) was multiplied by its PCSA (Cappellini et al., 2010; Maclellan et al., 2012). In addition, the activity of each spinal segment $S_{j}$ was multiplied by the estimated total number of motoneurons in this segment and divided by the largest number of MNs across segments (12,765 in L3; Table 1). The latter normalization has mainly an effect on the boundary segments L2 and S2 containing a total number of MNs considerably lower (two to three times) than the other segments we considered (Tomlinson and Irving, 1977).

Using the Kendall chart results in six rostrocaudal discrete activation waveforms, since the anatomical data are broken down into six segments (L2-S2). These waveforms were compared among the groups of subjects we studied. To visualize a continuous smoothed rostrocaudal spatiotemporal activation of the spinal cord, we also used a filled contour plot that computes isolines calculated from the activation waveform matrix and fills the areas between the isolines using separate colors.

To compare the general spatiotemporal features of the lumbosacral enlargement activation in different groups of subjects, and the relative activation of lumbar versus sacral segments in particular, we computed the timing of the maximal activation throughout the gait cycle and the ratio between the mean motoneuron activity in the dominant lumbar (sum of activity from L3 and L4) and sacral (sum of activity from S1 and S2) segments.

Assumptions. The basic analyses and interpretations of spinal maps are based on several assumptions (Grasso et al., 2004; Ivanenko et al., 2006): (1) motor pools are fairly stable in longitudinal spatial placement across individuals, in both children and adults, and through infant development to adulthood; (2) single motor pool activation is uniform, not spatially varied; (3) rectified EMG provides an indirect measure of the net firing rate of MNs of the corresponding muscle; and (4) temporal and spatial averaging does not obscure, but rather reveals, structuralfunctional changes through development.

The first assumption finds support in the studies on available myotomal charts (Romanes, 1951; Sharrard, 1964), though some minor anatomical deviations from the norm in single individuals have been documented (Phillips and Park, 1991; Stewart, 1992). We used the same available myotomal charts of muscle innervation for all age groups since the results derived from observations of the effects of root stimulation and root lesion in 2-days-old human neonates (Sharrard, 1964) agree (except for minor differences) with the findings derived from a study of the motor cell columns of the spinal cord (Sharrard, 1955) and with those obtained in adults (Kendall et al., 1993). The second assumption draws from the known Ia drive of motor pools, which is stereotypical and developmentally organized, independent of the normal pattern of activity early in development (Mendelson and Frank 1991; Chen et al., 2003). The third assumption implicit in all these analyses is that the rectified EMG provides an indirect measure of the net firing rate of MNs of that muscle (Yakovenko et al., 2002). Despite the simplification, this is a reasonable hypothesis because mean EMG increases almost linearly with the net motor unit firing rate (Hoffer et al., 1987; Day and Hulliger, 2001). As for the fourth assumption, individual EMGs may contain some additional or double bursts of activity in infants (see Figs. 3, 4A). Yet, the averaging procedure does not remove specific bursts of activity; it simply determines whether they are stable across subjects and within a step cycle. Such idiosyncratic EMG bursts are not necessarily devoid of function or completely independent of the pattern; rather, they may just not be sufficiently stable to contribute systematically to the overall muscle activation. In some cases, they may indeed be precursors of a toddler or adult pattern; however, they would escape our level of analysis. Our analysis focused on consistent and reproducible patterns of muscle activation in each group of subjects. The averaging procedure addresses that issue. However, the issue of individual variations will be addressed in Results (see Individual activation maps, below).

Finally, there is the interpretative issue of the relationship between segmental maps of motorneurons activation and the organization of the driving interneurons comprising the CPGs. Clearly, our results can only speak about the segmental motor pool activation, and only very indirect inferences can be drawn on CPGs. Indeed, at least at the cervical level of the spinal cord, data support a slightly different topography of driving interneurons compared to that of motor pools (Alstermark et al., 2007). We do not know how the drive sources for lumbosacral motor neurons are organized spatially in humans. Distinct populations of spinal interneurons could be involved in slow and fast speeds of locomotion (McLean et al., 2008). On the other hand, if premotor interneurons 


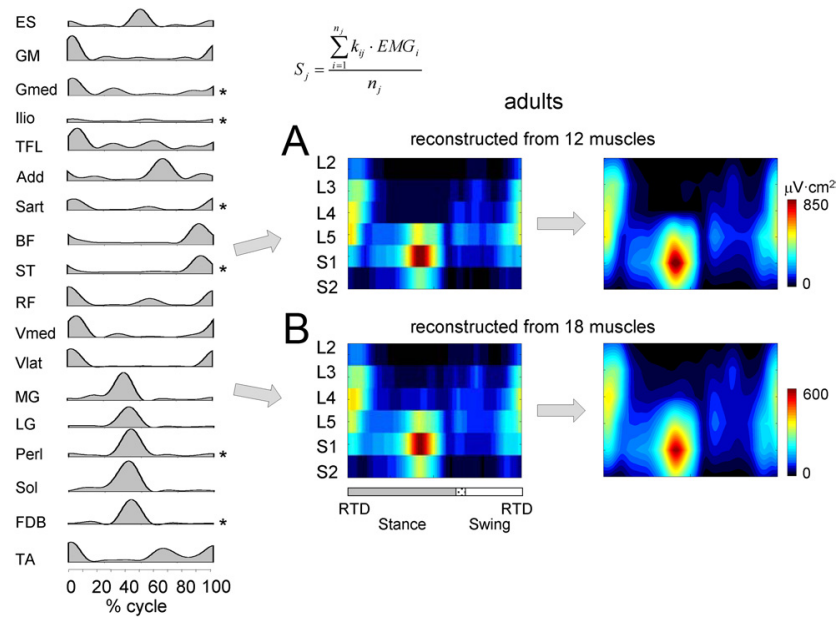

Figure 1. Reconstruction of spatiotemporal maps of motoneuron activity of the lumbosacral enlargement in adults walking at $5 \mathrm{~km} / \mathrm{h}$ using different sets of ipsilateral (right leg) EMG recordings. $\boldsymbol{A}$, Reconstructed from 18 muscles. $\boldsymbol{B}$, Reconstructed from 12 muscles (without ST, Gmed, Sart, llio, FDB, Perl muscles, marked by asterisks). Spinal activation maps were reconstructed using the normalized methods (normalized to the PCSA and to the total number of MNs in each segment; see Materials and Methods). Output segmental pattern is plotted in a color scale (in right panels, it was smoothed using a filled contour plot). The pattern is plotted versus normalized gait cycle. RTD, Right touchdown. Note similar spinal motor output patterns reconstructed from different sets of EMGs.

had a relatively simple and stereotyped topographic relationship with motoneurons, the motoneuron activity could be considered as an approximate readout of the interneuronal activity (O'Donovan et al., 2008). Moreover, according to some studies, motoneurons can be considered as integral elements of CPGs (Marder 1991; O’Donovan et al., 1998).

Sensitivity to the number of muscles included in the analysis. In the present study, practical considerations limited the set of muscles from which we could record in the neonates (see Materials and Methods). For the sake of comparison, we used the same set of muscles also in the other groups of subjects. Thus, there is the issue of how the specific selection of the muscles affects the resulting spatiotemporal maps of motoneuron activity. To verify the sensitivity of the results to the selected muscles, we used the EMG data recorded from a larger sample of muscles in adults (Ivanenko et al., 2008). Specifically, we compared the activation maps obtained by including all recorded muscles with those obtained by deleting an arbitrary subset of these muscles. In general, we found that the maps obtained by excluding any single one of the recorded muscles were similar to those obtained from the full set, presumably because the lumbosacral enlargement innervates numerous muscles and each muscle is innervated by several segments. In fact, even when using intramuscular recordings or when a slightly different set of muscle recordings was used to generate the maps during walking, both the maps and the basic bursts of the MN activity remained similar (Ivanenko et al., 2006, 2008). Figure 1 illustrates the method and its sensitivity to the set of muscles recorded. The spinal motor output was reconstructed using the EMG activity recorded in 10 adult subjects walking on a treadmill at $5 \mathrm{~km} / \mathrm{h}$ (Ivanenko et al., 2008). One can see that, despite some minor differences apparent in the color-scale maps, the segmental motor pool output estimated from the activity of 18 ipsilateral muscles [TA, Sol, MG, LG, RF, Vmed, Vlat, Add, ES, TFL, GM, peroneus longus (Perl), biceps femoris (BF; long head), semitendinosus (ST), flexor digitorum brevis (FDB), sartorius (Sart), iliopsoas (Ilio), and gluteus medius (Gmed)] is roughly similar to that estimated using 12 of these muscles (i.e., excluding ST, Gmed, Sart, Ilio, FDB, Perl). In particular, both estimates (Fig. $1 A, B$ ) capture four major loci of activity regardless of the number of muscles used: at the beginning of stance (lumbar activity), at the end of stance (sacral activity), at the onset of swing (L5 segment activity), and at the end of swing (both sacral and lumbar activity). In the present study, we used the set of muscles represented in Figure $1 A$ for all groups of subjects.
Potential effects of EMG cross talk. Another important concern in the present study is represented by the potential issue of cross talk. Activation maps are computed from many muscles and theoretically could be affected by electrical cross talk due to volume conduction of activity across adjacent muscles. The issue of cross talk is especially relevant in the case of neonates, given the small size of their body segments and the consequent close spacing of adjacent muscles. However, the small size of the EMG electrodes mainly used in our experiments and the interelectrode distance we used should have minimized the pickup from nearby muscles.

Nevertheless, we also addressed this issue by modeling the potential effects of different levels of cross talk in the EMG profiles. To this end, we used the data from adults because the maps in adults have been replicated in several previous studies (Grasso et al., 2004; Ivanenko et al., 2006, 2008; Cappellini et al., 2010; Monaco et al., 2010; Coscia et al., 2011; Maclellan et al., 2012). Here we reconstructed the spinal segmental output of adults by adding up incrementally the magnitude of cross talk. In particular, the following equations were implemented to simulate cross talk.

For the shank muscles we have the following:

$$
\begin{aligned}
\mathrm{EMG}_{\mathrm{Sol}} & =\mathrm{EMG}_{\mathrm{Sol}}+C \cdot \mathrm{EMG}_{\mathrm{TA}} ; \\
\mathrm{EMG}_{\mathrm{LG}} & =\mathrm{EMG}_{\mathrm{LG}}+C \cdot \mathrm{EMG}_{\mathrm{TA}} ; \\
\mathrm{EMG}_{\mathrm{MG}} & =\mathrm{EMG}_{\mathrm{MG}}+C \cdot \mathrm{EMG}_{\mathrm{TA}} ; \\
\mathrm{EMG}_{\mathrm{TA}}=\mathrm{EMG}_{\mathrm{TA}} & +C \cdot \frac{\mathrm{EMG}_{\mathrm{Sol}}+\mathrm{EMG}_{\mathrm{LG}}+\mathrm{EMG}_{\mathrm{MG}}}{3} .
\end{aligned}
$$

For the thigh muscles we have the following:

$$
\begin{array}{r}
\mathrm{EMG}_{\mathrm{Vlat}}=\mathrm{EMG}_{\mathrm{Vlat}}+C \cdot \mathrm{EMG}_{\mathrm{HS}}+C \cdot \mathrm{EMG}_{\mathrm{Add}}, \\
\mathrm{EMG}_{\mathrm{RF}}=\mathrm{EMG}_{\mathrm{RF}}+C \cdot \mathrm{EMG}_{\mathrm{HS}}+C \cdot \mathrm{EMG}_{\mathrm{Add}}, \\
\mathrm{EMG}_{\mathrm{Vmed}}=\mathrm{EMG}_{\mathrm{Vmed}}+C \cdot \mathrm{EMG}_{\mathrm{HS}}+C \cdot \mathrm{EMG}_{\mathrm{Add}}, \\
\mathrm{EMG}_{\mathrm{HS}}=\mathrm{EMG}_{\mathrm{HS}}+C \cdot \frac{\mathrm{EMG}_{\mathrm{Vlat}}+\mathrm{EMG}_{\mathrm{RF}}+\mathrm{EMG}_{\mathrm{Vmed}}}{3} \\
\mathrm{EMG}_{\mathrm{Add}}=\mathrm{EMG}_{\mathrm{Add}}+C \cdot \frac{\mathrm{EMG}_{\mathrm{Vlda}}+\mathrm{EMG}_{\mathrm{RF}}+\mathrm{EMG}_{\mathrm{Vmed}}}{3} \\
+C \cdot \mathrm{EMG}_{\mathrm{HS}}
\end{array}
$$

where $C$ indicates cross talk. The following levels of cross talk were modeled: 10,20 , and $30 \%(C=0.1,0.2$, and 0.3 , respectively). These levels of cross talk can be implicit in some EMG recordings (Dominici et al., 2011), taking into consideration the correlation coefficients $\left(r^{2}\right)$ between high-pass-filtered EMGs of flexors and extensors as suspect cross talk (Yang et al., 1998; Teulier et al., 2012). The results of this analysis will be presented in Figure 9.

\section{Statistics}

Descriptive statistics included the calculation of the mean and SD. The data analysis and spinal MN activity map construction were performed by means of custom software written in Matlab (version 7.12.0; Mathworks). A Pearson correlation coefficient was used to study the relationship between the age of preschoolers and the timing of lumbar activation, and between ensemble-averaged EMG waveforms in neonates stepping at different cadences. Paired $t$ tests were used to assess differences in the timing of the maximal activation of sacral and lumbar activation within each group of subjects. Unpaired $t$ tests were used to determine differences in the timing and relative activation levels of spinal segments in neonates with those of the other groups of subjects. Reported results are considered significant at $p<0.05$. 


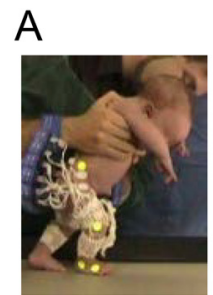

touchdown

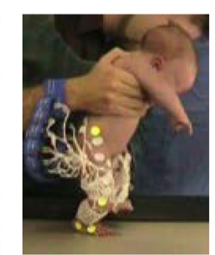

midstance

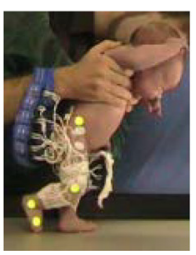

lift off

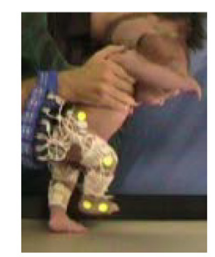

midswing

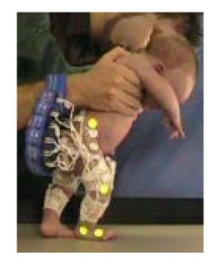

touchdown
B

six consecutive footprints in neonate
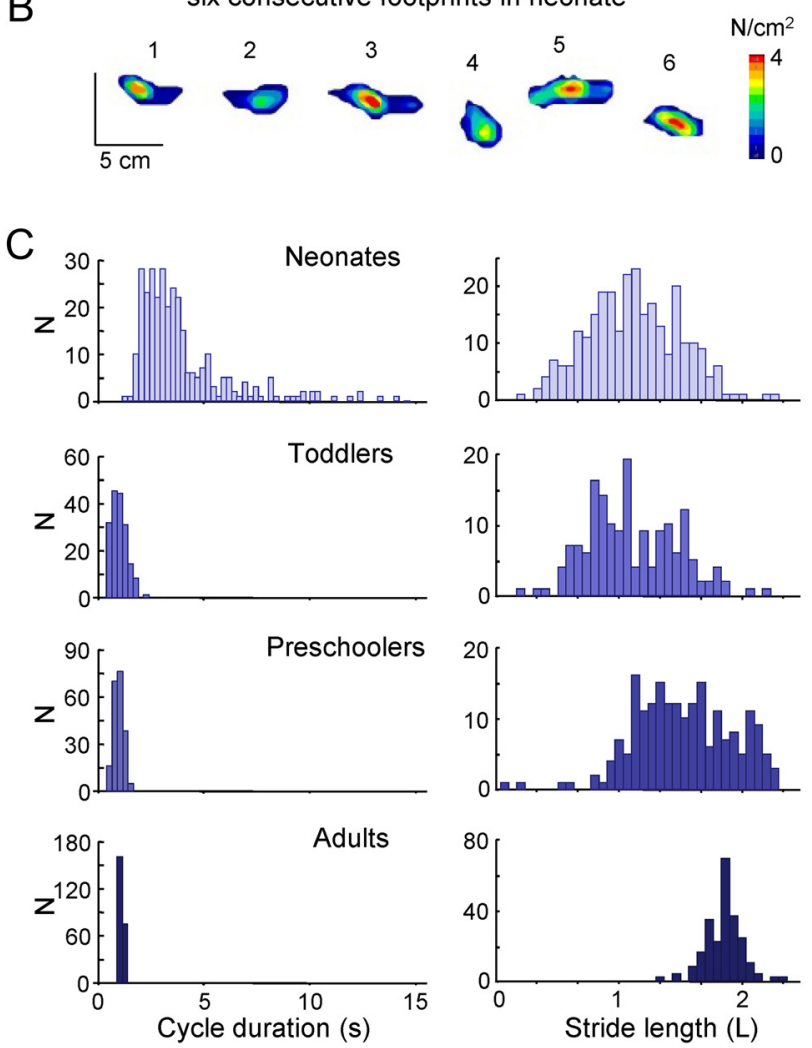

Figure 2. General gait parameters and kinematic patterns. $\boldsymbol{A}$, Illustration of a step cycle in a neonate. $\boldsymbol{B}$, Example of maximal plantar pressure distribution and corresponding limb loading in one neonate across six consecutive steps. $\boldsymbol{C}$, Distribution of individual cycle durations and stride lengths across all strides and subjects ( $n=356$ strides total) in neonates, toddlers, preschoolers, and adults. The stride length was normalized to the limb length $L$ (thigh plus shank) of the subjects.

\section{Results}

\section{Newborn stepping}

Infants can make walking-like steps when they are adequately supported ( $\sim 70 \%$ of body weight; $69 \pm 9 \%$ on average). The steps are generally irregular, and the cycle durations are much more variable than in the adult. The distribution of cycle duration and stride length across all steps and infants (Fig. $2 C$ ) peaks at values well above the adult ( $p<0.0001$, unpaired $t$ test). The variable footfall and limb loading patterns of Fig. $2 B$ are consistent with data reported previously in the literature (Forssberg, 1985; Yang et al., 1998; Okamoto et al., 2001, 2003).

In addition to the variability and long cycle duration, there are a few systematic features in the infant gait, including a bent knee and hip-flexed posture (Fig. 2A), and the absence of a clear heel strike (Fig. 2B). Some of these features persist at the onset of unsupported walking ( $~ 1$ year) (Forssberg, 1985; Ivanenko et al., 2005a), but they mature rapidly after a few months of independent walking experience (Ivanenko et al., 2007).

\section{EMG activity profiles}

Although the stepping movements of the neonates bear some rough resemblance to the walking movements of adults, the way in which they are controlled is quite different from the way adult walking is controlled. This is evident in the muscle activation profiles recorded in several different muscles (Fig. 3). Many of the leg muscles of the infant are coactivated over the ipsilateral stance phase, except for the TA and ES muscles, which are mostly active during ispilateral swing/contralateral stance (Forssberg, 1985; Yang et al., 1998; Okamoto et al., 2001, 2003). Almost all leg muscles are involved in stepping movements with alternate left/right coordination, while trunk muscles show typically only respiratory activity (with two to three bursts within each stride; Fig. $3 A$ ). It is worth noticing that respiratory activity of these muscles in adults is minute or absent entirely (Ivanenko et al., 2006). The duration of the activations is generally long, lasting about one-half of the cycle (Fig. $3 A, B)$. This is in marked contrast with the adult pattern of relatively brief activations occurring primarily at touchdown and liftoff (Fig. 3E). Although the ensemble profiles of activity in many muscles tended to be reproducible across neonate subjects (Fig. $3 A, B$ ), there was nevertheless considerable variability in the detailed time-varying structure of muscle activation both between infants and across step cycles (Fig. $4 A$ ). In particular, there was considerable phase structure and some double bursts in the EMG activity of some infants (Fig. 3B, HSr and TFLr). On the whole, however, the ensemble averages of infant EMG activity showed a quasisinusoidal modulation with the step cycle (Fig. 4A).

Given the wide distribution of gait cycle duration in neonates (Fig. 2C), it was necessary to determine whether the average EMG over a cycle could represent the activation for all cycle durations. Therefore we tested the normalization to gait cycle duration for the neonates by first dividing the neonate records into three groupings based on cycle duration (short, $<3$ s; medium, 3-4 s; long, $>4$ s) and then comparing the normalized EMGs across groups (Fig. $4 B)$. The results show that the normalized EMG activity profiles do not vary much with cycle duration. Thus, the mean correlation coefficient for EMGs between the shortest and longest step cycle was $0.90 \pm 0.08$ across all muscles (Fig. $4 B$ ).

\section{Spinal motoneuron activation maps}

We determined the average motoneuron activity pattern for each hemisegment of the spinal cord over the step cycle of each lower limb using the published data on the locations of motoneuron pools and the EMG activity profiles we recorded in individual muscles (see Materials and Methods). The motoneuron activation patterns for each age group are illustrated in Figure 5. 


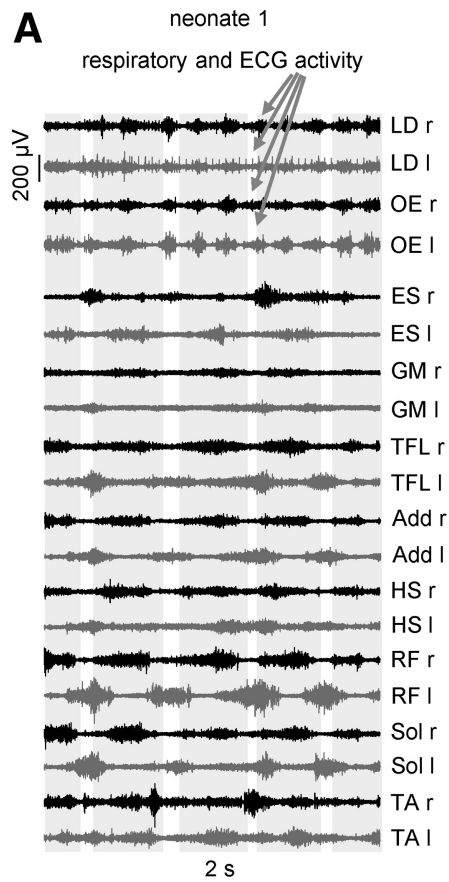

B

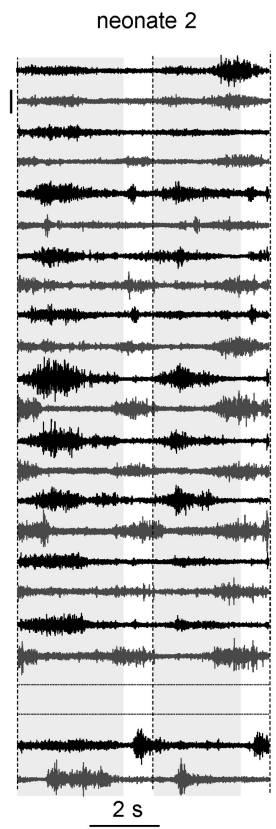

C

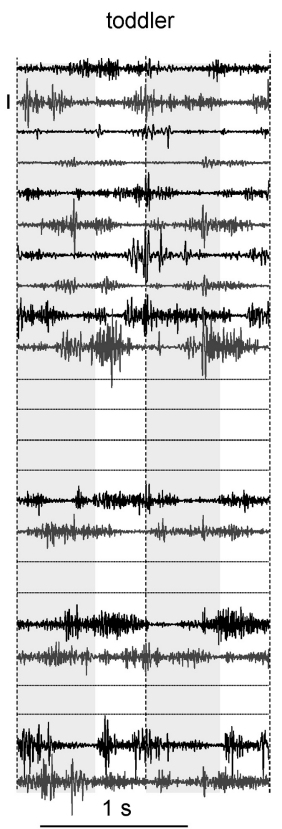

D

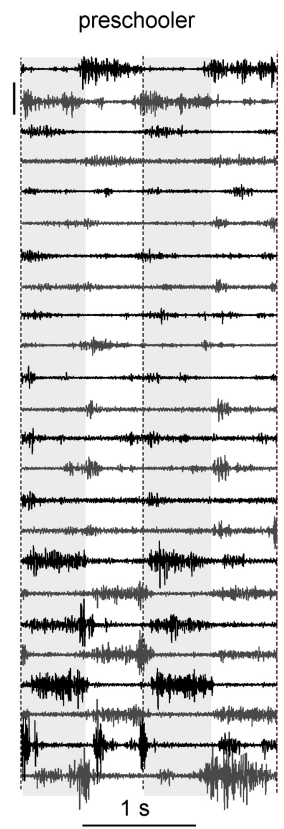

$\mathbf{E}$

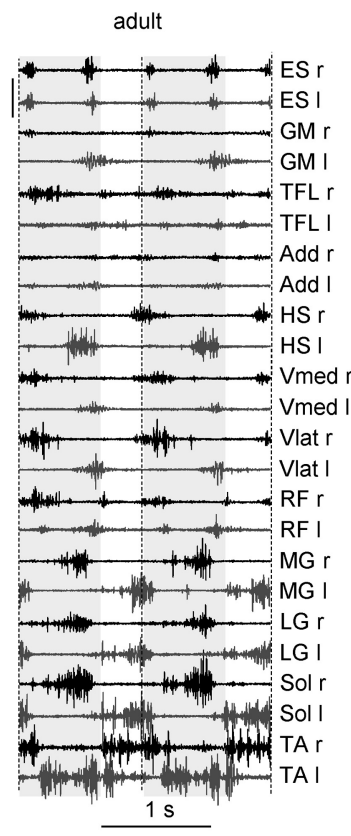

Figure 3. Examples of EMG traces during stepping in neonates, toddlers, preschoolers, and adults. $A$, Example of raw bilateral EMG traces in one neonate during four consecutive strides. Note a respiration-related activity in the $L D$ and 0 E muscles with a frequency $(\sim 0.9 \mathrm{~Hz})$ different from that of the gait cycle $(\sim 0.33 \mathrm{~Hz})$. Left $L D$ muscle demonstrated also a prominent electrocardiogramrelated activity. $\boldsymbol{B}-\boldsymbol{E}$, Examples of EMG traces during two consecutive strides in one neonate, toddler, preschooler, and adult. Horizontal lines for some muscles in neonate and toddler denote nonrecorded EMGs for these subjects. The stance phase of the right leg is evidenced by a shaded region. $r$, Right; I, left.

In Figure $5 A$, the maps are determined starting from the EMG amplitudes alone (Ivanenko et al., 2006; Cappellini et al., 2010). In Figure $5 B$, instead, the maps take into account both the number of motoneurons at each segmental level and the number of motor units in each muscle (see Materials and Methods). In both cases, the neonate pattern shows synchronous activation at all spinal levels that we considered (L2-S2); the activation is particularly prominent in the L3, L4, and S1 segments. These segments are activated mostly during the stance phase of the cycle, and symmetrically for the left and right sides of the spinal cord.

The corresponding activation pattern in the toddler becomes mainly focused in the sacral spinal segments at midstance, while a separate activation of both lumbar and sacral motoneurons occurs around touchdown. Preschoolers show a roughly similar pattern, but with a slightly greater separation between the activity at the beginning of stance and the rest of the pattern. The activation pattern of preschoolers appears to be a precursor closely related to the mature pattern seen in adults, where the activations are much shorter and with an evident separation of the distinct bursts.

\section{Individual activation maps}

The high interstep and interindividual variability of the EMG profiles in infants (Figs. 3, 4) may raise questions about spinal maps obtained from ensemble average data such as those plotted in Figure 5. Are they representative of the main activation patterns, or do they hide some basic pattern which is averaged out because of intertrial jitter? Thus, fine details of spatial "motor pool activation" such as the double bursts in Figure 3B may be obscured in maps derived from ensemble averages. To address this issue, we now consider the spatiotemporal maps computed in individual strides and subjects. Figure 6 shows some examples for all age groups. Despite the variability across steps, all neonates showed a systematic burst of activation, mainly expressed in lumbar segments, whose duration relative to the step cycle varied somewhat across individuals (Fig. 6A, compare neonate 1 , neonate 3 ). Intersubject variability was greater, however, in toddlers and preschoolers, presumably related to the longer span of ages included in these groups and the corresponding variability in maturation of the spinal patterns. Thus, activity in L3-L4 around touchdown was much more prominent in toddlers 2 and 3 than in toddler 1 . On the whole, however, the mean correlation coefficient between individuals (Fig. 6) and averaged (Fig. 5) segmental output waveforms was $0.85 \pm 0.13$. (This correlation coefficient was computed for each segment, and then the data for all segments were pooled together.) Thus, despite individual variations, the major features depicted in the averaged maps specific for each age (Fig. 5) are representative of the general trends in individual subjects.

\section{Quantitative developmental trends}

Figure 7 illustrates some quantitative features of the spatiotemporal activation of the lumbosacral enlargement in different groups of subjects and, in particular, the greater activation of lumbar (L3 and L4) versus sacral (S1 and S2) segments in neonates. On average, the dominant lumbar segments were significantly more active (relative to the sacral ones) in neonates compared to all other groups of subjects $(p<0.05$ in all cases, unpaired $t$ tests; Fig. $7 A$ ). The timing of the maximum of activity in lumbar segments was similar to that in sacral segments in neonates ( $p>0.8$, paired $t$ test), consistent with their quasisynchronous involvement, but the timing diverged in older children and adults (Fig. 7B). Thus, the timing of activity in lumbar segments of toddlers and older participants was significantly different from that in neonates, while the difference in timing in sacral segments started to be significantly different from that in neonates only in preschoolers and adults. However, due to the limited sample of participants and the variability in the data, we 

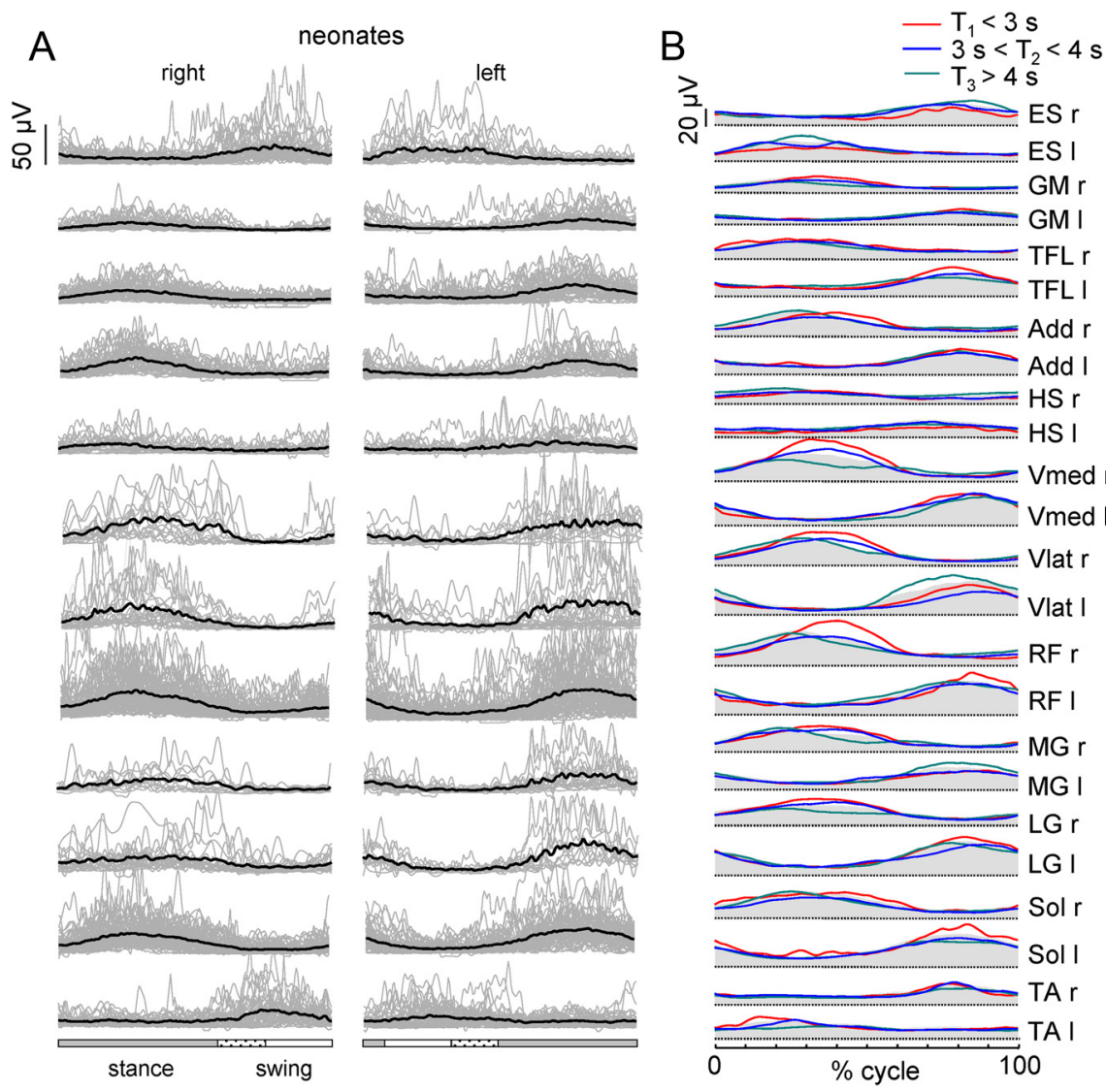

Figure 4. Effect of cycle duration on EMG profiles in neonates. A, Superimposed EMGs (gray color) of all neonates $(n=40)$ and all steps, independent of their duration. Ensemble-averaged EMGs for each muscle are shown in black color. As the relative duration of stance varied, a hatched region indicates an amount of variability in the stance phase duration across participants. $\boldsymbol{B}$, Ensembleaveraged (across all steps and neonates) EMG profiles for the left (I) and right ( $r$ ) legs are illustrated for the three intervals of different cycle durations: $\mathrm{T}_{1}=1.5-3 \mathrm{~s}(\mathrm{red}) ; \mathrm{T}_{2}=3-4 \mathrm{~s}$ (blue) and $\mathrm{T}_{3}=4-7 \mathrm{~s}$ (green). Ensemble-averaged EMGs across all steps (independent of their duration) are also shown by a gray shaded area. Note similar EMG profiles for different cycle durations.

cannot assess the time course of changes in the timing of activation on a more fine-grained scale.

Newborn stepping differs from more mature locomotion because it is quite slow, in addition to exhibiting several other differences in kinematics and kinetics (including the need for substantial support of body weight). An effect of walking speed on the basic EMG patterns has been demonstrated previously in adults (Ivanenko et al., 2004). Thus, it has been previously shown that the EMG activity bursts of adults are slightly but systematically delayed at lower walking speeds, in part related to a relatively longer stance duration, but the general structure of the locomotor program is roughly similar across speeds and body weight support conditions (Ivanenko et al., 2004). To verify the effect of stepping speed in neonates, we examined the spinal maps computed from the separate cycle-duration groupings of Figure $4 B$ and found no major differences other than a more uniform coactivation across lumbosacral segments during midstance at the lowest speeds (Fig. 8).

\section{Potential effects of EMG cross talk}

We addressed the issue of the potential impact of electrical cross talk on the spinal maps by using the EMG activity recorded in adults, whose spinal maps have been replicated in several previous studies (Grasso et al., 2004; Ivanenko et al., 2006, 2008; Cappellini et al., 2010; Monaco et al., 2010; Coscia et al., 2011; Maclellan et al., 2012). We reconstructed the spinal maps using the same procedures as before (Eq. 1), but with cross talk calculated based on a priori assumptions (Eq. 2-10; see Materials and Methods). Figure 9 illustrates the results of these simulations, where we assumed that Sol, LG, and MG are synergists and thus can produce similar cross-talk waveforms for TA. In a similar vein, we assumed that Vlat, RF, and Vmed are synergists and produce similar cross-talk waveforms for HS or Add. Regardless of the simulated source of cross talk, the basic results did not change appreciably if we assumed slightly different contributions of adjacent muscles. While the intensity and the width of the main loci of activations could be affected by adding cross talk (Fig. 9), this procedure did not give rise to the appearance of new loci of activation or to significant shifts of activity timings, nor did it result in a synchronous activation of lumbar and sacral segments typical of neonates (Figs. 5, 8), even when we considered $100 \%$ of cross talk (data not shown). Therefore, the critical hallmarks of neonate maps cannot be reproduced in the adult maps by simply assuming cross talk in the EMG recordings.

\section{Limitations of the analysis}

Even though the spatiotemporal activation maps are consistent across subjects and sets of muscle recordings (Figs. 1, 7,9) and were reproduced in several studies (Ivanenko et al., 2006, 2008; Cappellini et al., 2010; Monaco et al., 2010; Coscia et al., 2011; Maclellan et al., 2012), the applied method has limitations. For instance, some intrinsic thigh or foot muscles were not recorded. Nevertheless, their exclusion (e.g., flexor digitorum brevis) does not seem to jeopardize the major features of the segmental output (Fig. 1); moreover, the recorded muscles contribute a large part of the total cross-sectional area of leg muscles (Ward et al., 2009).

Also, the simulations (Fig. 9) showed that the main features of the neonate stepping could not be modeled by assuming cross talk in adult EMG recordings. In particular, the adult activation timing and sequencing were still present with simulated cross talk. While the activation was somewhat blurred or more intense with respect to the no-cross-talk condition (Fig. 9), there was still a separate lumbar activation followed by a sacral activation rather than the concurrent lumbosacral activation of the neonate.

The knee-bent posture, slower speed, and body weight support typical of neonate stepping may also influence the levels of activity (e.g., the timing of sacral activation may be somewhat delayed by the longer stance phase at the low speeds). Thus, one might question whether the specific motor patterns simply reflect differences in the biomechanics of stepping or these differences are the result of idiosyncratic CPG characteristics in neonates. Nevertheless, these factors unlikely account for the major differences in the developing human spinal cord (Fig. 5). For instance, the general timing pattern is conserved across different body weight support or walking speed conditions in adults (Ivanenko 
A
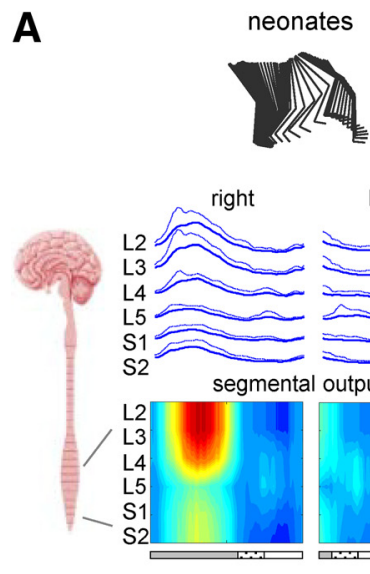

B
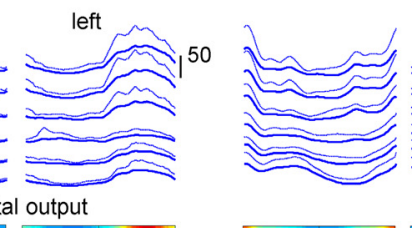

non-normalized method
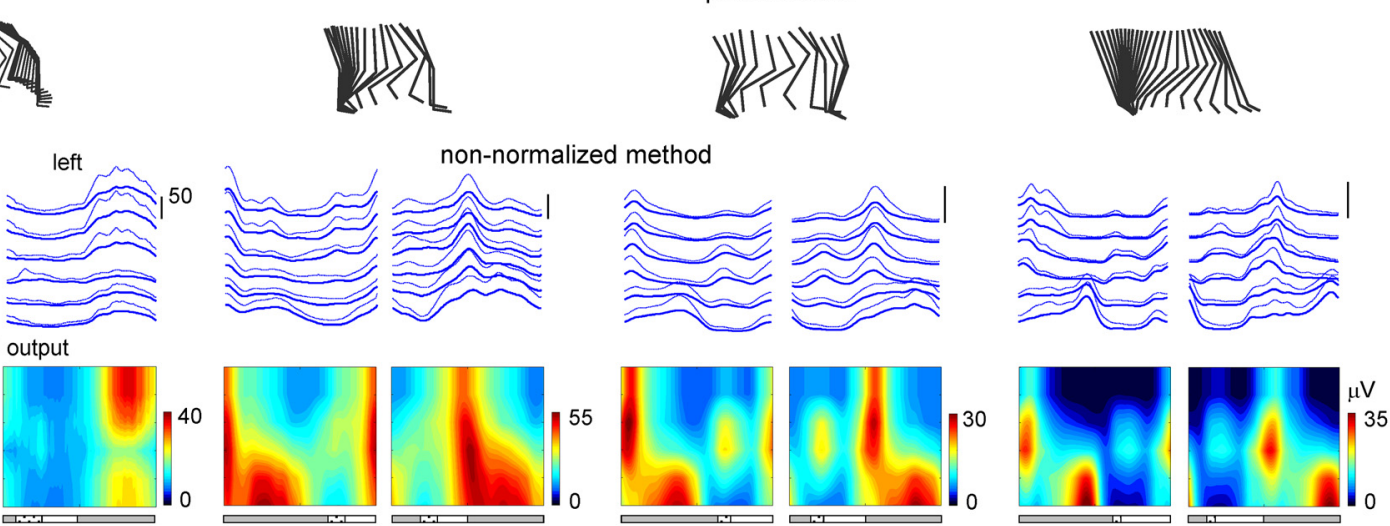

normalized method

(to PCSA and total number of MNs at each segment)
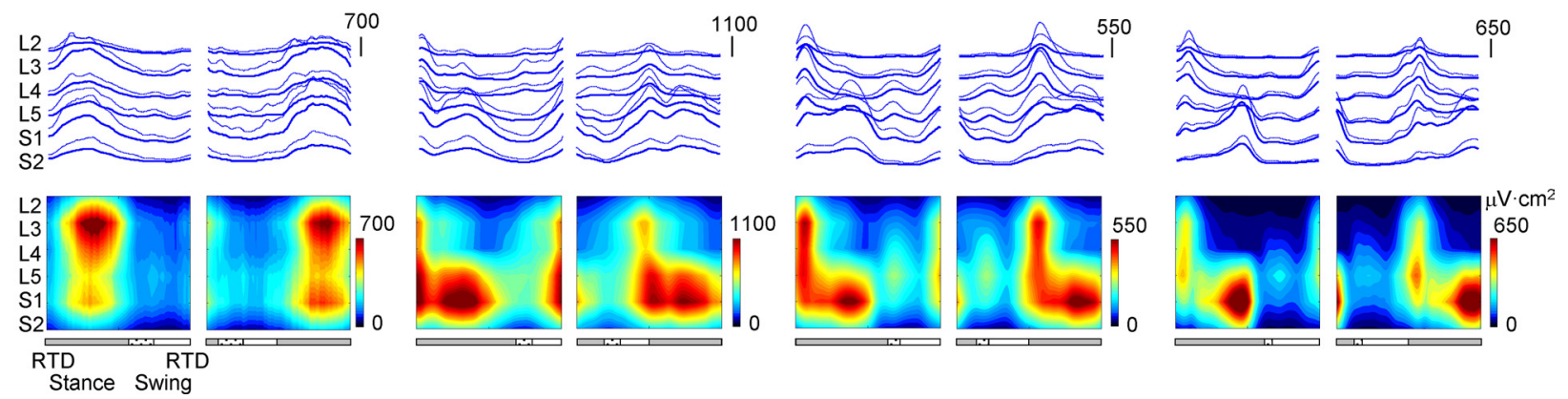

Figure 5. Spatiotemporal maps of motoneuron activity of the lumbosacral enlargement in neonates $(n=10)$, toddlers $(n=10)$, preschoolers $(n=10)$, and adults $(n=10)$. $\boldsymbol{A}, \boldsymbol{B}$, Spinal activation maps were reconstructed using both the nonnormalized method ( $\boldsymbol{A}$ ) and that normalized to the PCSA (Ward et al., 2009), and the total number of MNs in each segment (Tomlinson and Irving, 1977) (B). Output pattern of each segment is shown in the top panels (thick traces, means; thin traces, means +1 SD), while the same pattern is plotted in a color scale (using a filled contour plot) at the bottom. The pattern is plotted versus normalized gait cycle. RTD, Right touchdown. Note a quasi-sinusoidal output of all ventral roots in neonates.

et al., 2004). Also, the midstance activation of motoneurons in the neonate did not vary with speed or cycle duration (Fig. 8).

\section{Discussion}

Overall, the results revealed several features of the spinal motor output development in humans. The developmental trend involved a more selective and flexible control of the muscles (Figs. $3,5)$. The activation patterns in humans seem to be segregated by spinal segments in a specific manner and with a specific course of maturation (Figs. 5, 7).

\section{Postnatal development of locomotion and "stepping reflex" in neonates}

The human locomotion pattern shows several milestones during its development (Lacquaniti et al., 2012b). The stepping behavior in neonates, although present at birth, generally disappears for several months before toddler walking develops. There have been many debates about the origin and disappearance of the newborn stepping reflex such as the asynchronous maturation of cerebrospinal pathways and musculoskeletal plant (Thelen and Cooke, 1987). Newborn stepping may also be considered as an extension of rhythmic movements in "water" seen in utero (De Vries et al., 1984), especially taking into account that neonates are able to perform and learn swimming movements or to step supported by water buoyancy (Thelen and Cooke, 1987).

A remarkable feature of the motor output in neonates is the lack of foot-contact-related muscle activity, typical of toddler and adult walking. This could be explained at least in part by immature sensory and/or supraspinal modulation of stepping (Eyre et al., 2000; Yang and Gorassini, 2006). Indeed, in the absence of sensory modulation (e.g., during fictive motor tasks), the CPG circuitry tends to produce more sinusoidal-like patterns (Cuellar et al., 2009). Whatever the exact mechanism, the additional prominent activation at the onset of stance in the first months of life (Fig. 5) may imply a gradual structural reorganization of premotor drives (Hart and Giszter, 2010), the appearance of an additional layer in the CPG networks (McCrea and Rybak, 2008; Lacquaniti et al., 2012a), and/or more powerful descending and sensory influences on CPGs (Grillner, 2006; Kiehn, 2011). Also, longitudinal examination of spontaneous movements in 2- to 4-month-old infants showed a progression from a general activity involving all the limbs to an activity involving more selective interlimb coordination (Kanemaru et al., 2012).

\section{Maturation of segmental locomotor output in humans}

Motoneuron activation during locomotion is the end product of several processes. CPGs presumably generate the basic locomotor rhythm also in humans. There is also extensive feedback from various sensory receptors and supraspinal and intraspinal control for balance, direction, speed, and pathway. The development of the adult gait from the infant stepping is believed to represent the incorporation of these factors into locomotion control (Yang and Gorassini, 2006; Lacquaniti et al., 2012b). Our analysis of the segmental structure of motoneuron activity provides additional information about this developmental process. We observed a change from a uniform segmental control in both lumbar and sacral segments to a more segregated structure with separate lumbar and sacral activations (Fig. 5). 

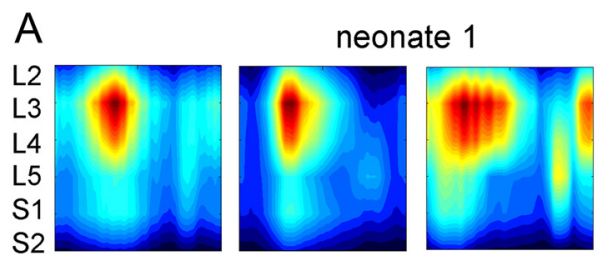

neonate 2
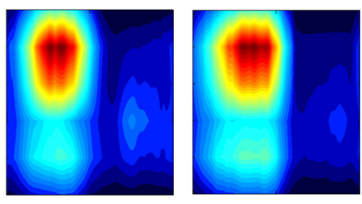

neonate 3

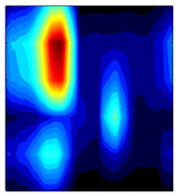

stride 1

stride 2

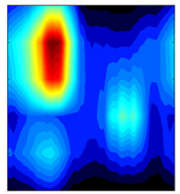

stride 3
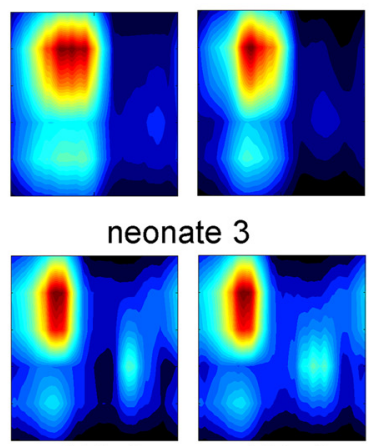
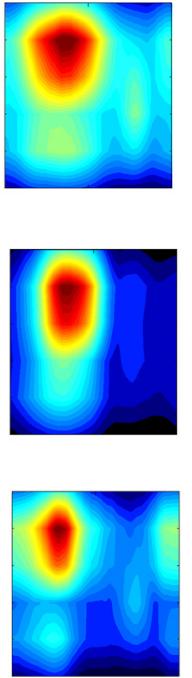

averaged across all trials
B
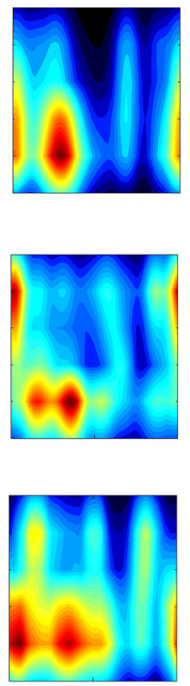

stride 1

D
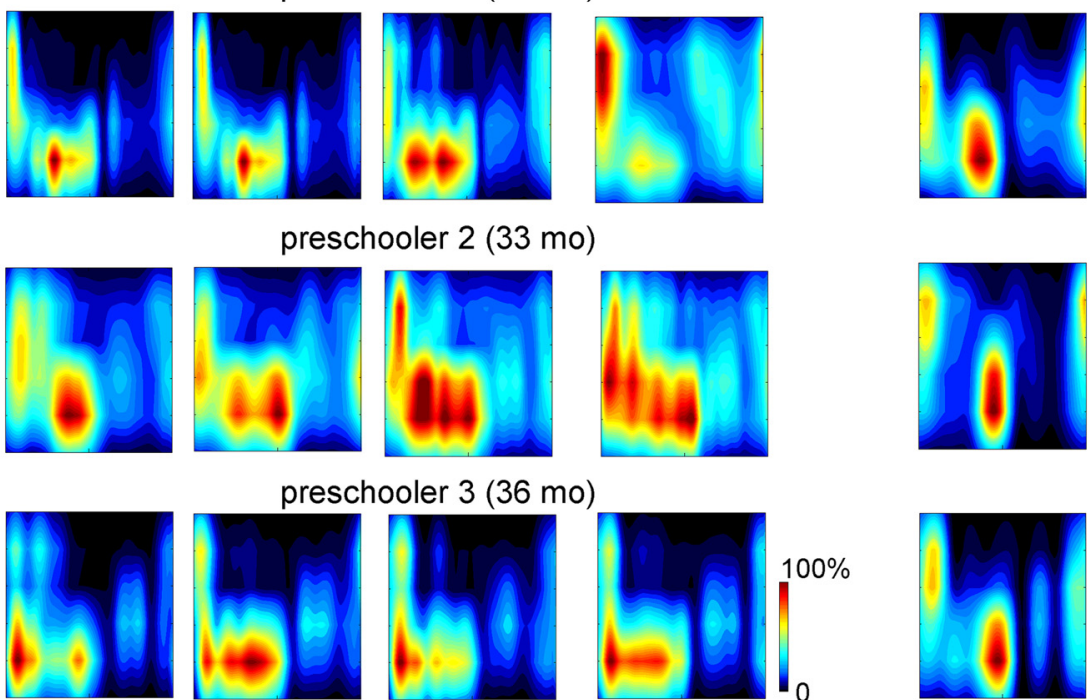

stride 1 preschooler 2 (33 mo)

preschooler 1 ( $25 \mathrm{mo})$
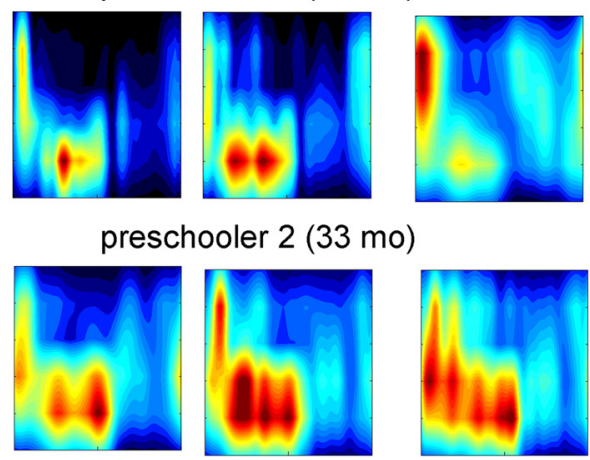

preschooler 3 (36 mo)

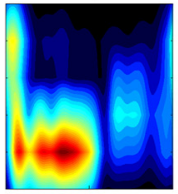

stride 2

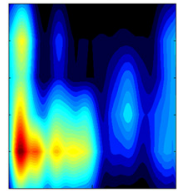

stride 3

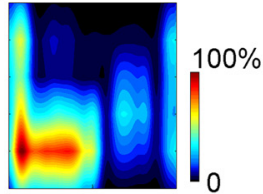

averaged cross all trials

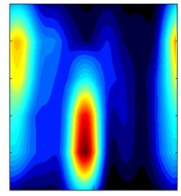

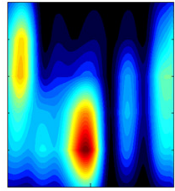

stride 1

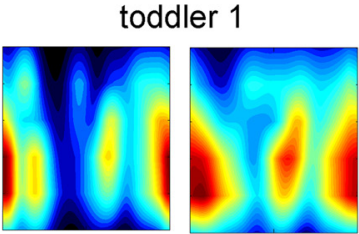

toddler 2

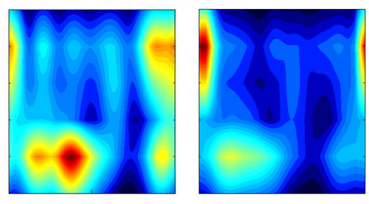

toddler 3

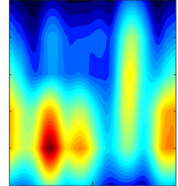

stride 2

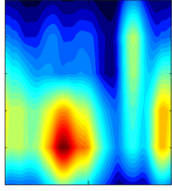

stride 3

adult 1
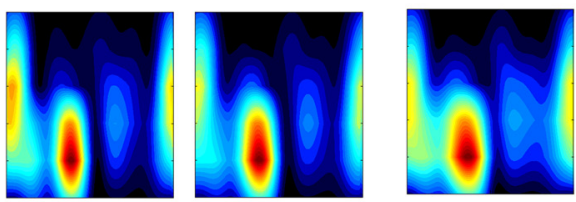

adult 2
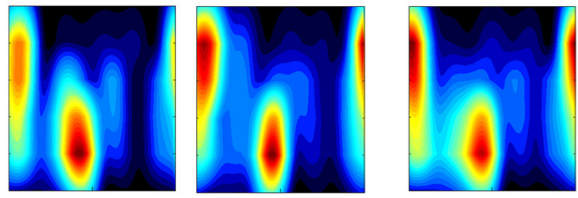

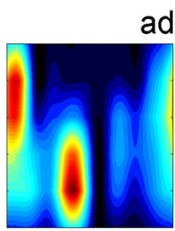

stride 2 adult 3

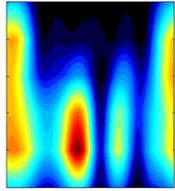

stride 3

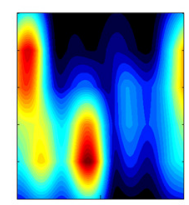

averaged across all trials

Figure 6. $\quad \boldsymbol{A}-\boldsymbol{D}$, Examples of spatiotemporal maps of motoneuron activity of the lumbosacral enlargement in three subjects for all age groups. For each individual, three individual strides and averaged stride (across all steps and trials, 5-20 strides) are shown. The same normalization as in Figure $5 B$ and the same color scale for all plots (100\% corresponds to the maximum activation to ease comparison between subplots) are shown. Note similar main features of the segmental output between individual and averaged (Fig. 5) spatiotemporal maps for all age groups.

The developmental changes in the spinal maps we observed between neonate and toddler included a shift in the predominant motoneuron activation in the stance phase from upper lumbar motoneurons in the neonate to the lower lumbar and sacral motoneurons in the toddler (Figs. 5, 7). This indicates a greater participation of the foot and lower extremity in support during stance, as the subjects are now supporting their entire body weight, and in producing propulsive or horizontal (shear) forces. In fact, even though infant stepping is irregular and with variable EMG patterns (Figs. 2C, 4A) (Okamoto and Okamoto, 2007; Teulier et al., 2012), there is an overall lack of foot-contact muscle activity in neonates (Figs. 4, 5, 8). Therefore, newborns typically exert vertical forces supporting part of their weight, but only tiny shear forces (Forssberg, 1985; Dominici et al., 2011; Lacquaniti et al., 2012b). Yet, the angular motion of the lower limb segments is already coordinated, resulting in a planar intersegmental covariance reminiscent of that seen in adults (Dominici et al., 2011). Thus, the reported features of the developing segmental motor output seem to be robust (Fig. 5), though it would be of great interest to relate this activity to different network circuitry components and how the sensory feedback and descending locomotor commands are integrated in the developing CPG.

\section{Comparative aspects}

The capacity of neural circuits to generate rhythmic activity in the absence of peripheral and supraspinal inputs is a welldocumented physiological phenomenon in animals (Orlovsky et al., 1999), and this potential most likely exists in the human spinal cord as well. Some elementary features of the vertebrate spinal locomotor network structure are preserved phylogenetically. The 

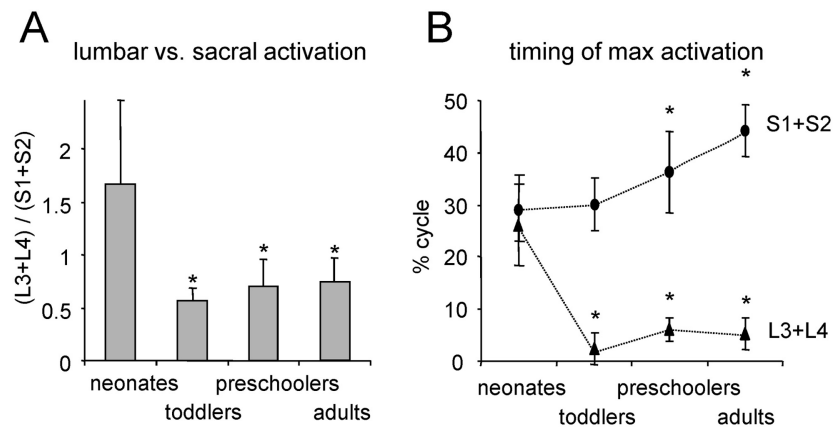

Figure 7. General features of lumbar and sacral segment output in different groups of subjects. $\boldsymbol{A}$, Relative mean activation of lumbar $(\mathrm{L} 3+\mathrm{L} 4)$ versus sacral $(\mathrm{S} 1+\mathrm{S} 2)$ segments. $\boldsymbol{B}$, Timing of their maximum activation. The values represent the mean $\pm S D(n=10$ for all groups of subjects). Asterisks denote significant differences with neonates (unpaired $t$ tests).
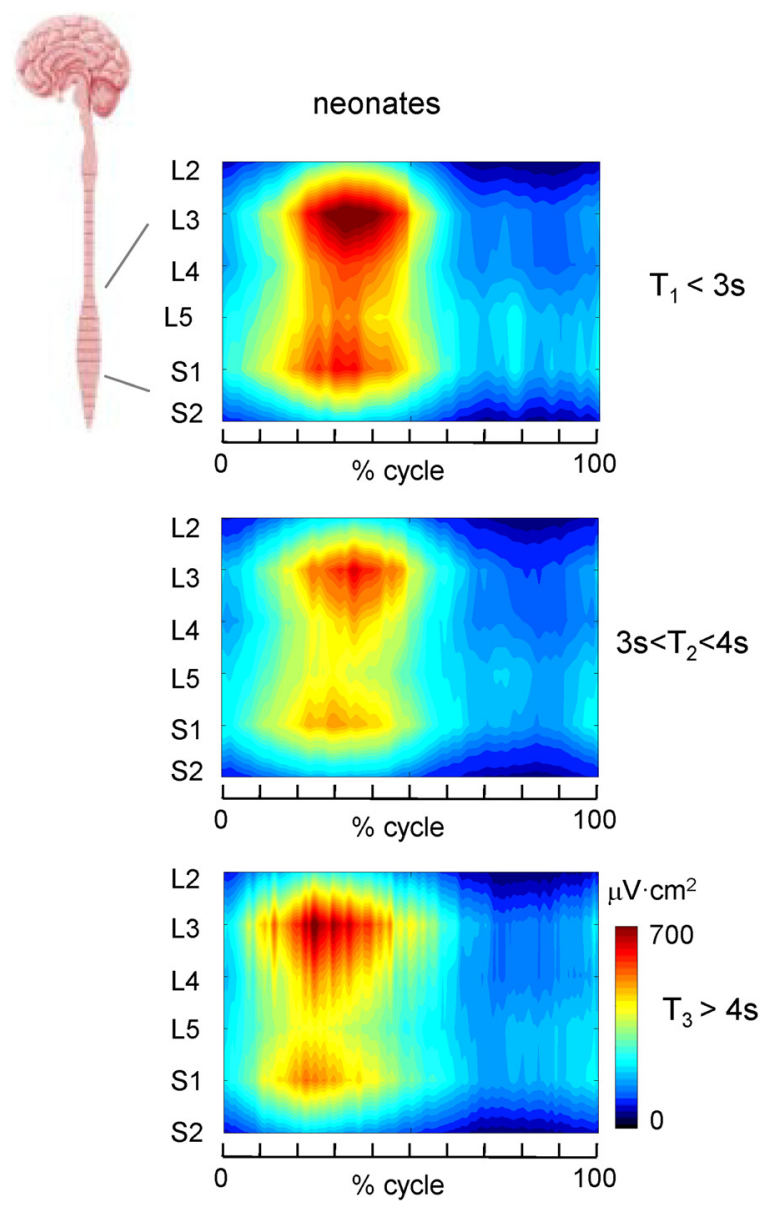

Figure 8. Effect of cycle duration on spatiotemporal patterns of segmental motor output in neonates $(n=10)$. Ensemble-averaged (across all steps and neonates, left and right leg data were pooled together; Fig. 4B) EMG profiles are mapped onto the known charts of segmental motoneuron localization in neonates and illustrated for the three intervals of different cycle durations: $T_{1}=1.5-3 \mathrm{~s}$ (top); $T_{2}=3-4 \mathrm{~s}$ (middle), and $T_{3}=4-7 \mathrm{~s}$ (bottom). Note similar patterns of activation for different cycle durations.

mammalian locomotor CPG is composed of multiple distributed rhythm-generating networks (Grillner, 1981, 2006, 2011) and includes excitatory neurons that are responsible for rhythm generation and glycinergic commissural interneurons that are directly involved in left/right alternation (Stein et al., 1998; Kiehn, 2006). Rostral lumbar segments (L1-L3 in rodents, L3-L5 in cats, D7-D10 in turtles) have a greater capacity to generate rhythmic

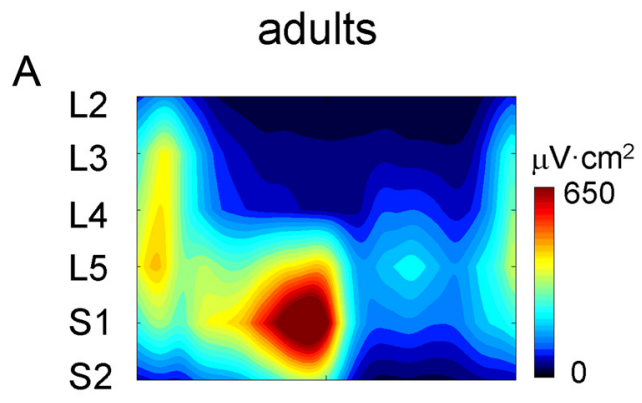

B
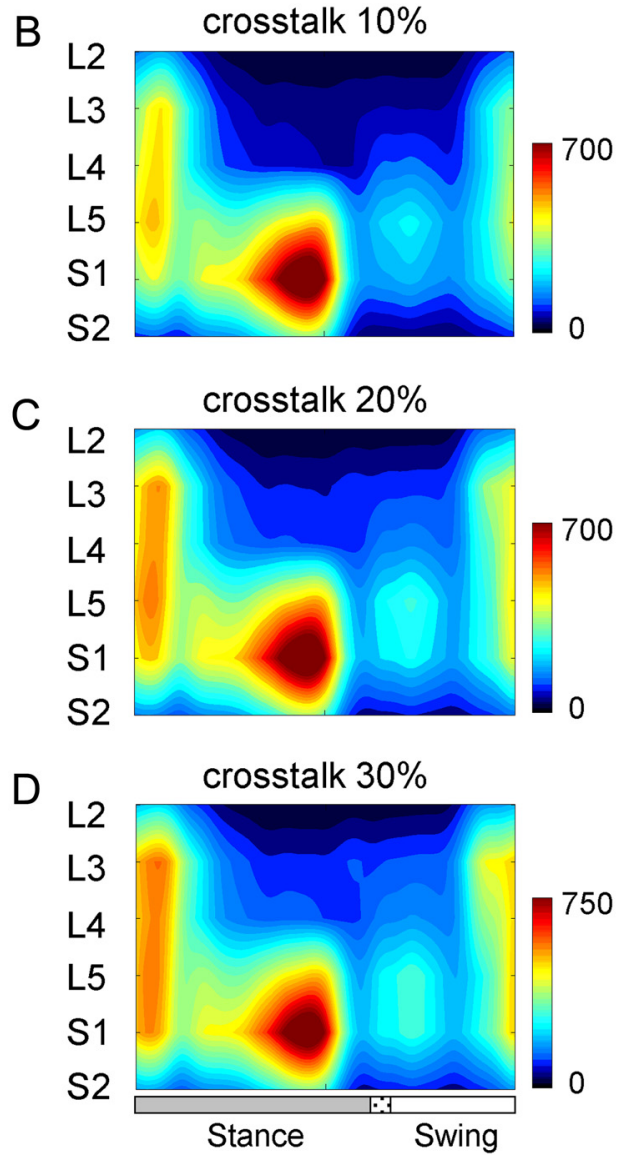

Figure 9. Parametric sensitivity of segmental motor output in adults to cross talk between flexors and extensors. $\boldsymbol{A}-\boldsymbol{D}$, Four levels of cross talk are illustrated: $0 \%(\boldsymbol{A}), 10 \%(\boldsymbol{B}), 20 \%(\boldsymbol{C})$, and $30 \%(\boldsymbol{D})$.

motor output in isolation than caudal segments (L4-L6, L6-S1, and S1-S2, respectively). Thus, the rhythmogenic capacity of the hindlimb CPG is distributed along the lumbar cord, but with a rostrocaudal excitability gradient (Deliagina et al., 1983; Mortin and Stein, 1989; Cazalets and Bertrand, 2000; Lev-Tov et al., 2000; Viney et al., 2002; Kiehn, 2006). The highest rhythmogenic capacity in the rostral cord where hip motor neurons are located suggests that rhythmogenic networks controlling hip movements act as a leading oscillator, entraining more caudal and less excitable oscillators, for example, those controlling the knee and ankle. Motor bursts propagate rostrally and caudally from the lumbar region to the most distant cord segments (Falgairolle and Cazalets, 2007). In humans, the upper lumbar pattern generator activity may also represent a major pacemaker (Shapkova and Schomburg, 2001; Gerasimenko et al., 2010; Harkema et al., 2011), whereas the sacral generator could play a subordinate role for adaptation to specific foot-support interactions (Selionov et al., 2009). 
In fact, the data suggest a developmental sequence that begins with a common patterning of motor pool activity in the entire lumbosacral spinal cord, proceeding to a separate patterning of activity in the lumbar and sacral spinal segments consistent with separate maturation of lumbar and sacral pattern generators in animals (Kremer and Lev-Tov, 1997; Cazalets and Bertrand, 2000; Lev-Tov et al., 2000; Vinay et al., 2002). In the perinatal rat, locomotion patterns seem to be largely driven by CPGs located in the upper lumbar and lower thoracic segments. With maturation, more localized patterns develop that are coupled to the sensory input. The human neonatal pattern of quasi-simultaneous activation throughout the lumbosacral spinal cord may reflect a similar unitary premotor drive to the lumbosacral motoneurons. The loss of this unitary drive is seen in the toddler with the appearance of separate lumbar and sacral activations corresponding respectively to touchdown and stance, and the need to provide support and balance. Thus the developmental features in the human and animal model may be sufficiently similar to provide an additional rationale for the use of these animal models to study human locomotion and its pathologies.

The mammalian quadrupedal pattern of development may have phases matching those of the human infant (Westerga and Gramsbergen, 1993; Dehorter et al., 2012). Mammalian quadrupeds are reported to progress from a lateral strut crawl to a parasagittal use of limbs as the corticospinal tract matures, and the cerebellum and cortex complete critical periods and establish motor maps. The timing of independent walking onset from conception in several different animals correlates with the mass of the brain, which in turn reflects the duration of its ontogenetic development (Garwicz et al., 2009). In addition, human gait has no analog in the animal kingdom and may imply an additional level or a particular organization of the CPG circuitry. In fact, despite its deceiving simplicity, human locomotion incorporating a heel strike and appropriate pendulum-like behavior of the center of body mass is a precise and complex motor task that requires learning (Dominici et al., 2007; Ivanenko et al., 2007). This depends on the specifics of the locomotor function, and the overall neural development and may account for the longer time to start walking in humans (Winter, 1989; Dominici et al., 2011).

\section{Summary and conclusions}

Given some assumptions described in Materials and Methods, we conclude from our results that the development of human locomotion from the neonate to the toddler involves a partial reorganization of the spinal circuitry. A striking feature of newborn stepping is a rostrocaudal coactivation of motoneurons in the lumbosacral cord but with a higher overall activation of lumbar versus sacral segments, consistent with a rostrocaudal excitability gradient. The lumbosacral coactivation of motoneurons seen in the neonate is no longer apparent in the toddler when the lumbar and sacral motoneurons assume separate activation patterns. The separation becomes more prominent with further development with progressively shorter motoneuron activations.

\section{References}

Alstermark B, Isa T, Pettersson LG, Sasaki S (2007) The C3-C4 propriospinal system in the cat and monkey: a spinal pre-motoneuronal centre for voluntary motor control. Acta Physiol (Oxf) 189:123-140. CrossRef

Bosma R, Stroman PW (2012) Diffusion tensor imaging in the human spinal cord: development, limitations, and clinical applications. Crit Rev Biomed Eng 40:1-20. CrossRef Medline

Cappellini G, Ivanenko YP, Poppele RE, Lacquaniti F (2006) Motor patterns in human walking and running. J Neurophysiol 95:3426-3437. CrossRef Medline
Cappellini G, Ivanenko YP, Dominici N, Poppele RE, Lacquaniti F (2010) Migration of motor pool activity in the spinal cord reflects body mechanics in human locomotion. J Neurophysiol 104:3064-3073. CrossRef Medline

Cazalets JR, Bertrand S (2000) Ubiquity of motor networks in the spinal cord of vertebrates. Brain Res Bull 53:627-634. CrossRef Medline

Chen HH, Hippenmeyer S, Arber S, Frank E (2003) Development of the monosynaptic stretch reflex circuit. Curr Opin Neurobiol 13:96-102. CrossRef Medline

Coscia M, Monaco V, Capogrosso M, Chisari C, Micera S (2011) Computational aspects of $\mathrm{MN}$ activity estimation: a case study with post-stroke subjects. IEEE Int Conf Rehabil Robot 2011:5975405. Medline

Cuellar CA, Tapia JA, Juárez V, Quevedo J, Linares P, Martínez L, Manjarrez E (2009) Propagation of sinusoidal electrical waves along the spinal cord during a fictive motor task. J Neurosci 29:798-810. CrossRef Medline

Day SJ, Hulliger M (2001) Experimental simulation of cat electromyogram: evidence for algebraic summation of motor-unit action-potential trains. J Neurophysiol 86:2144-2158. Medline

Dehorter N, Vinay L, Hammond C, Ben-Ari Y (2012) Timing of developmental sequences in different brain structures: physiological and pathological implications. Eur J Neurosci 35:1846-1856. CrossRef Medline

Deliagina TG, Orlovsky GN, Pavlova GA (1983) The capacity for generation of rhythmic oscillations is distributed in the lumbosacral spinal cord of the cat. Exp Brain Res 53:81-90. Medline

De Vries JIP, Visser GHA, Prechtl HFR (1984) Fetal motility in the first half of pregnancy. In: Continuity of neural functions from prenatal to postnatal life. Clinics in Developmental Medicine, Vol 94 (Prechtl HFR, ed), pp 46-64. Oxford: Spastics International Medical.

Dominici N, Ivanenko YP, Lacquaniti F (2007) Control of foot trajectory in walking toddlers: adaptation to load changes. J Neurophysiol 97:2790-2801. CrossRef Medline

Dominici N, Ivanenko YP, Cappellini G, Zampagni ML, Lacquaniti F (2010) Kinematic strategies in newly walking toddlers stepping over different support surfaces. J Neurophysiol 103:1673-1684. CrossRef Medline

Dominici N, Ivanenko YP, Cappellini G, d'Avella A, Mondì V, Cicchese M, Fabiano A, Silei T, Di Paolo A, Giannini C, Poppele RE, Lacquaniti F (2011) Locomotor primitives in newborn babies and their development. Science 334:997-999. CrossRef Medline

Eyre JA, Miller S, Clowry GJ, Conway EA, Watts C (2000) Functional corticospinal projections are established prenatally in the human foetus permitting involvement in the development of spinal motor centres. Brain 123:51-64. CrossRef Medline

Falgairolle M, Cazalets JR (2007) Metachronal coupling between spinal neuronal networks during locomotor activity in newborn rat. J Physiol 580: 87-102. CrossRef Medline

Forssberg H (1985) Ontogeny of human locomotor control. I. Infant stepping, supported locomotion and transition to independent locomotion. Exp Brain Res 57:480-493. Medline

Garcia-Campmany L, Stam FJ, Goulding M (2010) From circuits to behaviour: motor networks in vertebrates. Curr Opin Neurobiol 20:116-125. CrossRef Medline

Garwicz M, Christensson M, Psouni E (2009) A unifying model for timing of walking onset in humans and other mammals. Proc Natl Acad Sci U S A 106:21889-21893. CrossRef Medline

Gerasimenko Y, Gorodnichev R, Machueva E, Pivovarova E, Semyenov D, Savochin A, Roy RR, Edgerton VR (2010) Novel and direct access to the human locomotor spinal circuitry. J Neurosci 30:3700-3708. CrossRef Medline

Giove F, Garreffa G, Giulietti G, Mangia S, Colonnese C, Maraviglia B (2004) Issues about the fMRI of the human spinal cord. Magn Reson Imaging 22:1505-1516. CrossRef Medline

Goulding M (2009) Circuits controlling vertebrate locomotion: moving in a new direction. Nat Rev Neurosci 10:507-518. CrossRef Medline

Grasso R, Ivanenko YP, Zago M, Molinari M, Scivoletto G, Castellano V, Macellari V, Lacquaniti F (2004) Distributed plasticity of locomotor pattern generators in spinal cord injured patients. Brain 127:1019-1034. CrossRef Medline

Grillner S (1981) Control of locomotion in bipeds, tetrapods, and fish. In: Handbook of physiology, Section 1, The nervous system, Motor control, Vol 2, Part 1 (Brookhart JM, Mountcastle VB, eds), pp 1179-1236. Bethesda, MD: American Physiological Society.

Grillner S (2006) Biological pattern generation: the cellular and computa- 
tional logic of networks in motion. Neuron 52:751-766. CrossRef Medline

Grillner S (2011) Neuroscience. Human locomotor circuits conform. Science 334:912-913. CrossRef Medline

Harkema S, Gerasimenko Y, Hodes J, Burdick J, Angeli C, Chen Y, Ferreira C, Willhite A, Rejc E, Grossman RG, Edgerton VR (2011) Effect of epidural stimulation of the lumbosacral spinal cord on voluntary movement, standing, and assisted stepping after motor complete paraplegia: a case study. Lancet 377:1938-1947. CrossRef Medline

Hart CB, Giszter SF (2010) A neural basis for motor primitives in the spinal cord. J Neurosci 30:1322-1336. CrossRef Medline

Hoffer JA, Sugano N, Loeb GE, Marks WB, O’Donovan MJ, Pratt CA (1987) Cat hindlimb motoneurons during locomotion. II. Normal activity patterns. J Neurophysiol 57:530-553. Medline

Ivanenko YP, Poppele RE, Lacquaniti F (2004) Five basic muscle activation patterns account for muscle activity during human locomotion. J Physiol 556:267-282. Medline

Ivanenko YP, Dominici N, Cappellini G, Lacquaniti F (2005a) Kinematics in newly walking toddlers does not depend upon postural stability. J Neurophysiol 94:754-763. CrossRef Medline

Ivanenko YP, Cappellini G, Dominici N, Poppele RE, Lacquaniti F (2005b) Coordination of locomotion with voluntary movements in humans. J Neurosci 25:7238-7253. CrossRef Medline

Ivanenko YP, Poppele RE, Lacquaniti F (2006) Spinal cord maps of spatiotemporal alpha-motoneuron activation in humans walking at different speeds. J Neurophysiol 95:602-618. Medline

Ivanenko YP, Dominici N, Lacquaniti F (2007) Development of independent walking in toddlers. Exerc Sport Sci Rev 35:67-73. Medline

Ivanenko YP, Cappellini G, Poppele RE, Lacquaniti F (2008) Spatiotemporal organization of alpha-motoneuron activity in the human spinal cord during different gaits and gait transitions. Eur J Neurosci 27:3351-3368. CrossRef Medline

Jessell TM, Sürmeli G, Kelly JS (2011) Motor neurons and the sense of place. Neuron 72:419-424. CrossRef Medline

Kanemaru N, Watanabe H, Taga G (2012) Increasing selectivity of interlimb coordination during spontaneous movements in 2- to 4-month-old infants. Exp Brain Res 218:49-61. CrossRef Medline

Kendall FP, McCreary EK, Provance PG (1993) Muscles: testing and function. Baltimore: Lippincott, Williams and Wilkins.

Kiehn O (2006) Locomotor circuits in the mammalian spinal cord. Annu Rev Neurosci 29:279-306. CrossRef Medline

Kiehn O (2011) Development and functional organization of spinal locomotor circuits. Curr Opin Neurobiol 21:100-109. CrossRef Medline

Kremer E, Lev-Tov A (1997) Localization of the spinal network associated with generation of hindlimb locomotion in the neonatal rat and organization of its transverse coupling system. J Neurophysiol 77:1155-1170. Medline

Lacquaniti F, Ivanenko YP, Zago M (2012a) Patterned control of human locomotion. J Physiol 590:2189-2199. CrossRef Medline

Lacquaniti F, Ivanenko YP, Zago M (2012b) Development of human locomotion. Curr Opin Neurobiol 22:822-828. CrossRef Medline

Lev-Tov A, Delvolvé I, Kremer E (2000) Sacrocaudal afferents induce rhythmic efferent bursting in isolated spinal cords of neonatal rats. J Neurophysiol 83:888-894. Medline

Maclellan MJ, Ivanenko YP, Cappellini G, Sylos Labini F, Lacquaniti F (2012) Features of hand-foot crawling behavior in human adults. J Neurophysiol 107:114-125. Medline

Marder E (1991) Modifiability of pattern generator. Curr Opin Neurobiol 1:571-576. CrossRef Medline

McCrea DA, Rybak IA (2008) Organization of mammalian locomotor rhythm and pattern generation. Brain Res Rev 57:134-146. CrossRef Medline

McLean DL, Masino MA, Koh IY, Lindquist WB, Fetcho JR (2008) Continuous shifts in the active set of spinal interneurons during changes in locomotor speed. Nat Neurosci 11:1419-1429. CrossRef Medline

Mendelson B, Frank E (1991) Specific monosynaptic sensory-motor connections form in the absence of patterned neural activity and motoneuronal cell death. J Neurosci 11:1390-1403. Medline

Monaco V, Ghionzoli A, Micera S (2010) Age-related modifications of muscle synergies and spinal cord activity during locomotion. J Neurophysiol 104:2092-2102. CrossRef Medline

Mortin LI, Stein PS (1989) Spinal cord segments containing key elements of the central pattern generators for three forms of scratch reflex in the turtle. J Neurosci 9:2285-2296. Medline

O’Donovan MJ, Wenner P, Chub N, Tabak J, Rinzel J (1998) Mechanisms of spontaneous activity in the developing spinal cord and their relevance to locomotion. Ann N Y Acad Sci 860:130-141. CrossRef

O'Donovan MJ, Bonnot A, Mentis GZ, Arai Y, Chub N, Shneider NA, Wenner P (2008) Imaging the spatiotemporal organization of neural activity in the developing spinal cord. Dev Neurobiol 68:788-803. CrossRef Medline

Okamoto T, Okamoto K (2007) Development of gait by electromyography. Application to gait analysis and evaluation. Osaka, Japan: Walking Development Group.

Okamoto T, Okamoto K, Andrew PD (2001) Electromyographic study of newborn stepping in neonates and young infants. Electromyogr Clin Neurophysiol 41:289-296. Medline

Okamoto T, Okamoto K, Andrew PD (2003) Electromyographic developmental changes in one individual from newborn stepping to mature walking. Gait Posture 17:18-27. CrossRef Medline

Orlovsky GN, Deliagina TG, Grillner S (1999) Neural control of locomotion. From mollusc to man. Oxford, UK: Oxford UP.

Patla AE, Calvert TW, Stein RB (1985) Model of a pattern generator for locomotion in mammals. Am J Physiol 248:R484-R494. Medline

Patrick SK, Noah JA, Yang JF (2009) Interlimb coordination in human crawling reveals similarities in development and neural control with quadrupeds. J Neurophysiol 101:603-613. Medline

Phillips LH 2nd, Park TS (1991) Electrophysiologic mapping of the segmental anatomy of the muscles of the lower extremity. Muscle Nerve 14:12131218. CrossRef Medline

Romanes GJ (1951) The motor cell columns of the lumbo-sacral spinal cord of the cat. J Comp Neurol 94:313-363. CrossRef Medline

Selionov VA, Ivanenko YP, Solopova IA, Gurfinkel VS (2009) Tonic central and sensory stimuli facilitate involuntary air-stepping in humans. J Neurophysiol 101:2847-2858. CrossRef Medline

Shapkova EY, Schomburg ED (2001) Two types of motor modulation underlying human stepping evoked by spinal cord electrical stimulation (SCES). Acta Physiol Pharmacol Bulg 26:155-157. Medline

Sharrard WJ (1955) The distribution of the permanent paralysis in the lower limb in poliomyelitis. J Bone Joint Surg 37:540-558. Medline

Sharrard WJ (1964) The segmental innervation of the lower limb muscles in man. Ann R Coll Surg Engl 35:106-122. Medline

Stein PS, McCullough ML, Currie SN (1998) Spinal motor patterns in the turtle. Ann N Y Acad Sci 860:142-154. CrossRef

Stewart JD (1992) Electrophysiological mapping of the segmental anatomy of the muscles of the lower extremity. Muscle Nerve 15:965-966. Medline

Sürmeli G, Akay T, Ippolito GC, Tucker PW, Jessell TM (2011) Patterns of spinal sensory-motor connectivity prescribed by a dorsoventral positional template. Cell 147:653-665. CrossRef Medline

Teulier C, Sansom JK, Muraszko K, Ulrich BD (2012) Longitudinal changes in muscle activity during infants' treadmill stepping. J Neurophysiol 108: 853-862. CrossRef Medline

Thelen E, Cooke DW (1987) Relationship between newborn stepping and later walking: a new interpretation. Dev Med Child Neurol 29:380-393. Medline

Tomlinson BE, Irving D (1977) The numbers of limb motor neurons in the human lumbosacral cord throughout life. J Neurol Sci 34:213-219. CrossRef Medline

Vinay L, Brocard F, Clarac F, Norreel JC, Pearlstein E, Pflieger JF (2002) Development of posture and locomotion: an interplay of endogenously generated activities and neurotrophic actions by descending pathways. Brain Res Brain Res Rev 40:118-129. Medline

Ward SR, Eng CM, Smallwood LH, Lieber RL (2009) Are current measurements of lower extremity muscle architecture accurate? Clin Orthop Relat Res 467:1074-1082. CrossRef Medline

Warp E, Agarwal G, Wyart C, Friedmann D, Oldfield CS, Conner A, Del Bene F, Arrenberg AB, Baier H, Isacoff EY (2012) Emergence of patterned activity in the developing zebrafish spinal cord. Curr Biol 22:93-102. CrossRef Medline

Westerga J, Gramsbergen A (1993) Development of locomotion in the rat: the significance of early movements. Early Hum Dev 34:89-100. CrossRef Medline

Winter DA (1989) Biomechanics of normal and pathological gait: implica- 
tions for understanding human locomotor control. J Mot Behav 21:337355. Medline

Winter DA, Yack HJ (1987) EMG profiles during normal human walking: stride-to-stride and inter-subject variability. Electroencephalogr Clin Neurophysiol 67:402-411. CrossRef Medline

Yakovenko S, Mushahwar V, VanderHorst V, Holstege G, Prochazka A (2002) Spatiotemporal activation of lumbosacral motoneurons in the locomotor step cycle. J Neurophysiol 87:1542-1553. Medline
Yang JF, Gorassini M (2006) Spinal and brain control of human walking: implications for retraining of walking. Neuroscientist 12:379-389. CrossRef Medline

Yang JF, Stephens MJ, Vishram R (1998) Infant stepping: a method to study the sensory control of human walking. J Physiol 507:927-937. CrossRef Medline 